\title{
DIGITAL CANOPY MODEL ESTIMATION FROM TANDEM-X INTERFEROMETRY USING HIGH-RESOLUTION LIDAR DEM
}

\author{
Maciej J. Soja ${ }^{1)}$ and Lars M. H. Ulander ${ }^{1,2)}$ \\ ${ }^{1)}$ Chalmers University of Technology, Gothenburg, Sweden \\ ${ }^{2)}$ Swedish Defence Research Agency, Linköping, Sweden
}

\begin{abstract}
Interferometric TanDEM-X data are used together with high-resolution, airborne lidar-derived digital elevation models (DEMs) to produce digital canopy models (DCMs) for the boreal forests of Remningstorp and Krycklan, situated in southern and northern Sweden, respectively. An overview of interferometric data processing is given. First results showing the potential of TanDEM-X-based forest canopy mapping are presented. It is concluded that baselines giving height-of-ambiguity values in the order of 50-80 meters are preferable, although factors such as angle of incidence and along-track baseline are also of importance. Clear-cuts can easily be detected thanks to the high resolution of TanDEM-X imagery. Seasonal variations of scattering height are most visible for deciduous trees, where the scattering height is significantly lower in the winter, probably due to the lack of leaves.
\end{abstract}

Index Terms - synthetic aperture radar (SAR), digital canopy model (DCM), forest mapping

\section{INTRODUCTION}

TanDEM-X (TDX) is a new X-band (9.65 GHz) synthetic aperture radar (SAR) satellite from the German Aerospace Center (DLR) launched in June 2010. TanDEM-X circulates in Earth's orbit in a tight tandem formation with its almost identical twin satellite TerraSAR-X (TSX), launched in June 2007. Together, the two satellites create the first permanent, single-pass SAR interferometer in space (here simply called TDM). The main goal of the TDM mission is to obtain the first global, high-resolution digital elevation model (DEM) [1].

In densely forested regions, X-band signals will primarily scatter from tree canopies, thus introducing a significant offset from ground level in TDM-based DEM measurements. However, if an accurate, high-resolution external DEM is available, this offset can be estimated and used for forest height mapping.

The main scope of this paper is to show that digital canopy models (DCMs) can be derived from TDM interferometry using an accurate, high-resolution external DEM. Two test sites are used: Remningstorp, situated in southern Sweden, and Krycklan, situated in northern Sweden. TDM-derived DCMs are here compared to lidar DCMs acquired during the BioSAR 2008 and 2010 campaigns. Similar studies were performed in [2], where XSAR data from the SRTM InSAR mission were used, and in [3], where airborne X-band ESAR data were used to measure forest height.

This paper is structured as follows. First, the experimental and reference data used in this study are presented. Next, the processing steps for TDM DCM generation are summarized. Thereafter, a comparison of TDM DCMs and lidar DCMs is shown, and some observations are made. The work is then summarized and evaluated in the last section.

\section{EXPERIMENTAL DATA}

In this study, interferometric TDM image pairs are used to create digital canopy models (DCM) for two test sites in Sweden: Remningstorp, a hemi-boreal forest situated in southern Sweden, and Krycklan, a boreal forest situated in northern Sweden. While the topography in Remningstorp is fairly flat, Krycklan features a hilly terrain with stand-level slopes reaching 20 degrees. Both test sites have been extensively used in previous studies (see [4] and references therein); large quantities of reference data are thus available. Interferometric TDM image pairs provided by the German Aerospace Center for both Remningstorp and Krycklan are used.

As ground level reference, high-resolution lidar DEMs are used. They were acquired during an on-going nationwide lidar mapping campaign run by Swedish Land Survey. The campaign aims at covering the whole of Sweden with a $2 \mathrm{~m} \times 2 \mathrm{~m}$ grid DEM with a mean height error of the order of 0.5 meters. The campaign is planned to be finished by the year 2015.

The reference lidar DCMs used in this study were acquired within the BioSAR campaigns (in 2010 for Remningstorp and in 2008 for Krycklan). Stand delineations are also used. For Remningstorp, a set of 665 delineated regions is available, consisting primarily of forest stands, but 
also pastures, marshes, open fields, water, private properties, and others. For Krycklan, a set of 1372 delineated regions is available, consisting primarily of mature and young forest stands, but also bare rock, marshes, private properties, and others. For both test sites, meteorological data provided by Swedish Hydrological and Meteorological Institute (SMHI) including temperature, precipitation, and wind information are also available.

\section{DCM PROCESSING}

TDM data are provided by DLR in CoSSC (Co-registered Slant range Single look Complex) format. For the data acquired in the bistatic mode, two images are available - one monostatic image acquired by the active satellite (master image), and one bistatic image acquired by the passive satellite (slave image). As the two TDM images are already co-registered and filtered to the same Doppler spectrum [5], no additional pre-processing is required and interferometric processing can be carried out directly.

TDM data are processed interferometrically using a Matlab algorithm based on [5] and developed specially for this project. The processing can be summarized in the following five steps:

1. Georeferencing and DEM interpolation,

2. DEM interferogram computation,

3. TDM interferogram flattening,

4. DCM computation,

5. DCM geometric correction.

During the first step, range (R) and zero-Doppler time (ZDT) are computed for each DEM pixel using a NewtonRhapson-based algorithm. A look-up table (LUT) mapping DEM coordinates to range-ZDT coordinates is created. The LUT is then re-sampled to the range-ZDT grid of the master image, giving a geocoding LUT containing DEM coordinates for each SLC pixel. This inversion is the single most computationally demanding step in the whole processing chain. When the geocoding LUT is created, DEM data can easily be interpolated to radar geometry.

In the second step, DEM phase is computed using satellite state vectors (orbit position vectors). The quasibistatic acquisition geometry has to be considered in this step. In practice, the difference between the bistatic range (distance from the master satellite to a DEM pixel and then back to the slave satellite) and the monostatic range (twoway distance between the master satellite and a DEM pixel) is computed, and then transformed into a synthetic DEM interferogram. Also here, a Newton-Rhapson based algorithm is used to compute the exact monostatic and bistatic range values. The displacements of the satellites during pulse propagation time are also accounted for.

During the third step, the DEM phase is removed from the TDM interferogram by means of complex multiplication of the TDM interferogram with the complex conjugate of the synthetic DEM interferogram. The interferogram is then multilooked coherently and the phase is unwrapped using an optimal $2 \pi$-window which minimizes the risk of wrapping. In practice, this is done by a multiplication with a complex constant with unit magnitude and a phase chosen in such a way, that the unwrapped phase histogram has its minima at 0 and $2 \pi$.

In the fourth step, interferometric phase is transformed into forest height using a linear function. The slope of the function is equal to the height-of-ambiguity (HOA) divided by $2 \pi$, while the intercept is an image-specific height offset. HOA is the vertical height shift corresponding to a $2 \pi$ interferogram phase shift, and it is computed geometrically from orbit state vectors and DEM, see [1]. The height offset is chosen in such a way, that the mean TDM height in a sample of non-forested areas is zero. The non-forested areas used as reference are chosen from the delineated regions classified as open fields and pastures, with an additional restriction on lidar DCM being lower than $0.1 \mathrm{~m}$.

In the final fifth step, the DCMs are geometrically corrected to better match the lidar maps. As SAR images are acquired in side-looking geometry, there is a range offset present if scatterer position deviates from the DEM. This offset is compensated through range resampling and interpolation. This step is not mandatory, but it improves the geometric agreement between lidar DCM and TDM DCM, especially in the borders between regions with different land use or forest characteristics.

Note that all interferometric processing is performed in SLC (radar) geometry rather than in DEM geometry to preserve the high resolution of the data, minimize the need for oversampling, and preserve the original frequency components. Processing in DEM geometry would be simpler to implement and would not require the computationally demanding LUT inversion, but it is not recommended due to the aforementioned problems. Stand-level averages were also computed in SLC geometry. Only the final DCM products were re-sampled to DEM geometry for better viewing and comparison with reference data. All figures shown in this paper are in UTM coordinates (zone 33N for Remningstorp and zone 34N for Krycklan). Polarization dependence is not studied in this paper. All DCMs presented in this work were derived from VV-polarized TDM data.

\section{RESULTS}

A comparison between TDM DCM and lidar DCM is shown in Figure 1 for a $1500 \mathrm{~m}$ x $1200 \mathrm{~m}$ region in Remningstorp. The three TDM DCMs were acquired at three different baselines corresponding to different HOA values. As it can be observed, image quality decreases when HOA increases. HOA is inversely proportional to the perpendicular baseline. An increased HOA means lower baseline, which leads to a decreased height sensitivity. The signal-to-noise ratio in phase images becomes lower. Large baselines are therefore desirable for interferometric forest imaging. However, too 

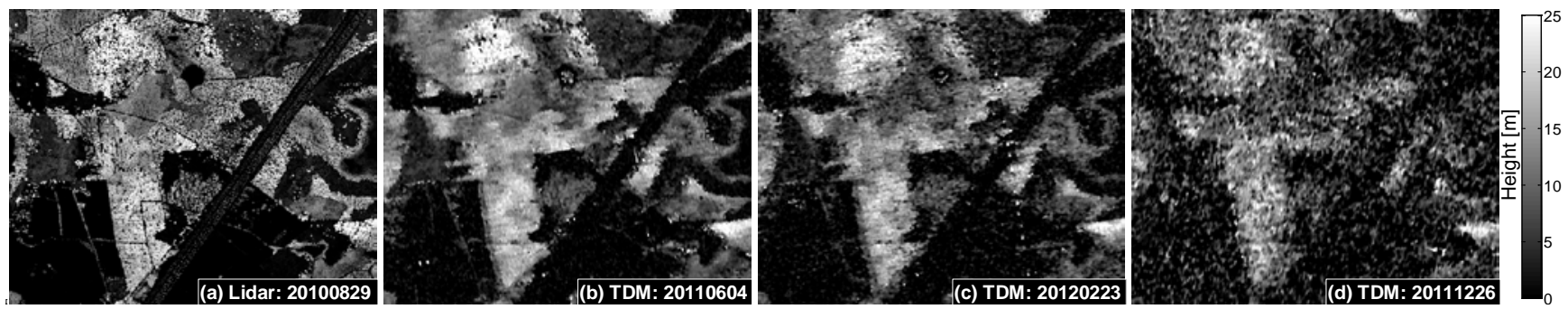

Figure 1 A comparison between four DCMs for a $1500 \mathrm{~m}$ x $1200 \mathrm{~m}$ region in Remningstorp is here shown: a) Lidar DCM from August 2010, b) TDM DCM from June 2011 with HOA=49 m and $\theta_{0}=41^{\circ}$, c) TDM DCM from February 2012 with HOA=77 m and $\theta_{0}=41^{\circ}$, and d) TDM DCM from December 2011 with HOA=178 m and $\theta_{0}=34^{\circ}$. Clearly, noise level in TDM DCMs increases with HOA. VV-polarized TDM data were used for TDM DCM estimation. $\theta_{0}$ is the nominal angle of incidence.

(a) HOA comparison: small difference

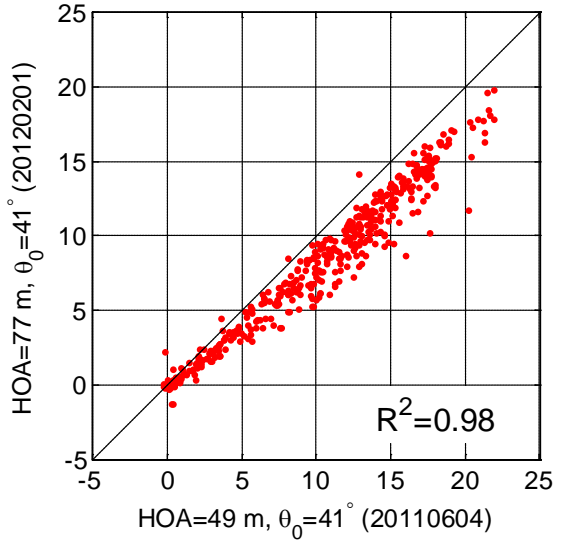

(d) Temporal stability: low HOA

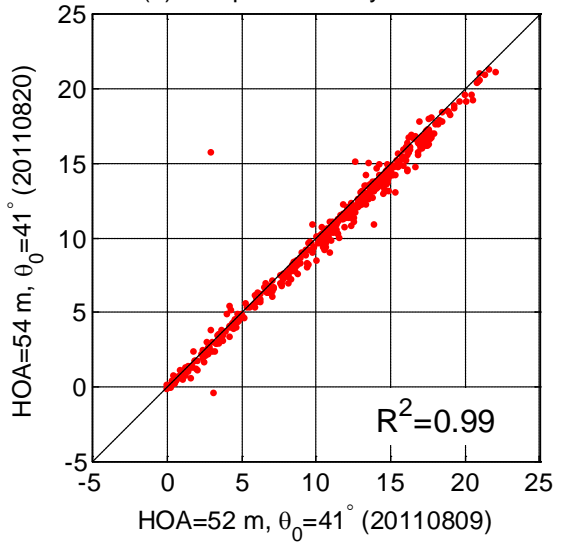

(c) HOA comparison: medium difference



(e) Temporal stability: medium HOA

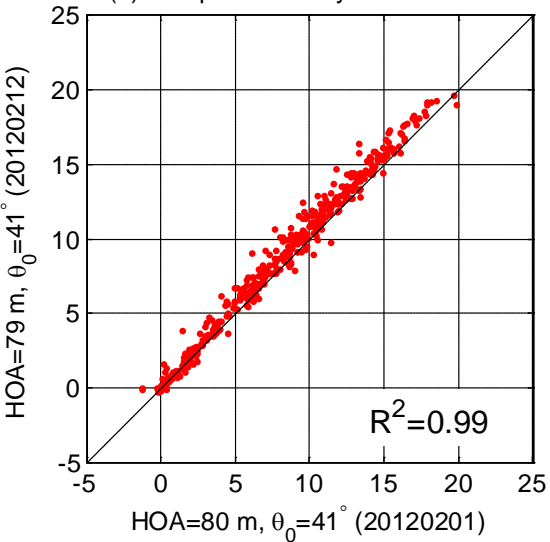

(b) HOA comparison: large difference

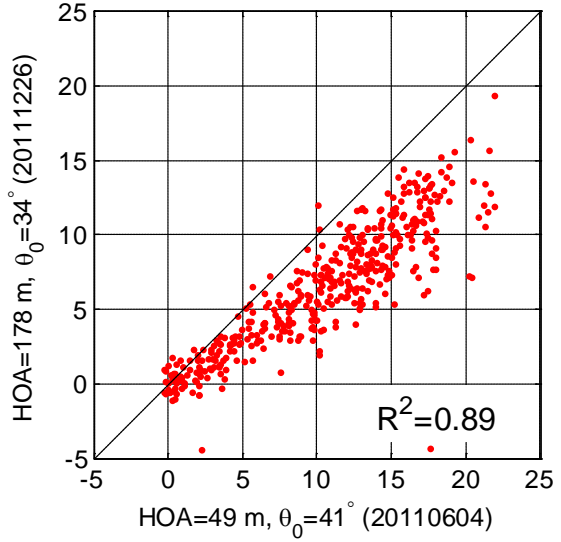

(f) Temporal stability: high $\mathrm{HOA}$

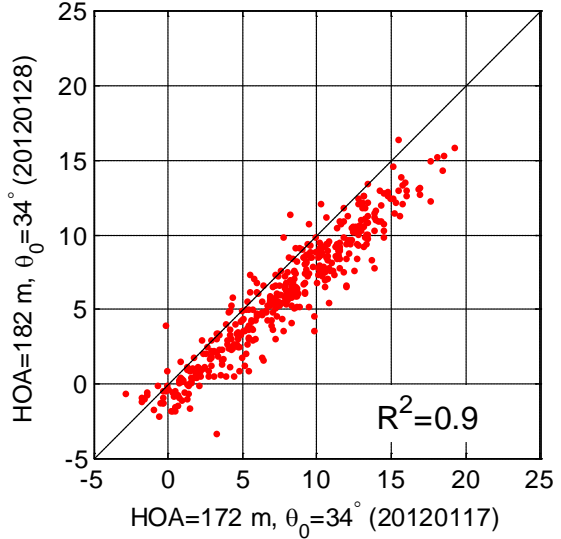

Figure 2 Scatter plots showing the consistency of stand-level height estimates (in meters) for 429 delineated forest regions in Remningstorp in different acquisition scenarios are shown above. Only VV-polarized acquisitions over Remningstorp were used. The dates of the acquisitions are shown in parentheses. $\theta_{0}$ is the nominal angle of incidence.
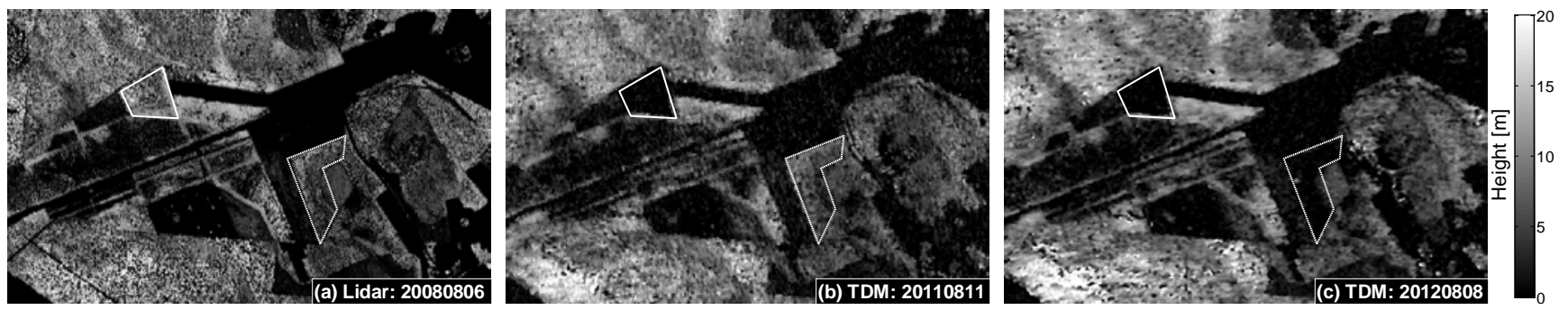

Figure 3 A comparison between three DCMs for a $1600 \mathrm{~m}$ x $1000 \mathrm{~m}$ region in Krycklan is here shown: a) Lidar DCM from August 2008, b) TDM DCM from August 2011 with HOA=56 m, and c) TDM DCM from August 2012 with HOA=38 m. Two clear-cuts have been delineated: one clear-cut made between August 2008 and August 2011 is delineated with solid lines, while a second clear-cut made between August 2011 and August 2012 is delineated with dashed lines. VV-polarized TDM data acquired at the nominal angle of incidence of $41^{\circ}$ were used for TDM DCM estimation. 
large baselines lead to strong baseline decorrelation and an increased risk of wrapping. It has been observed, that HOA values between 50 and 80 meters present a good compromise. Note that, although HOA is a good indicator of the potential quality of an image, other parameters such as the angle of incidence, along-track baseline, and weather conditions can be of importance.
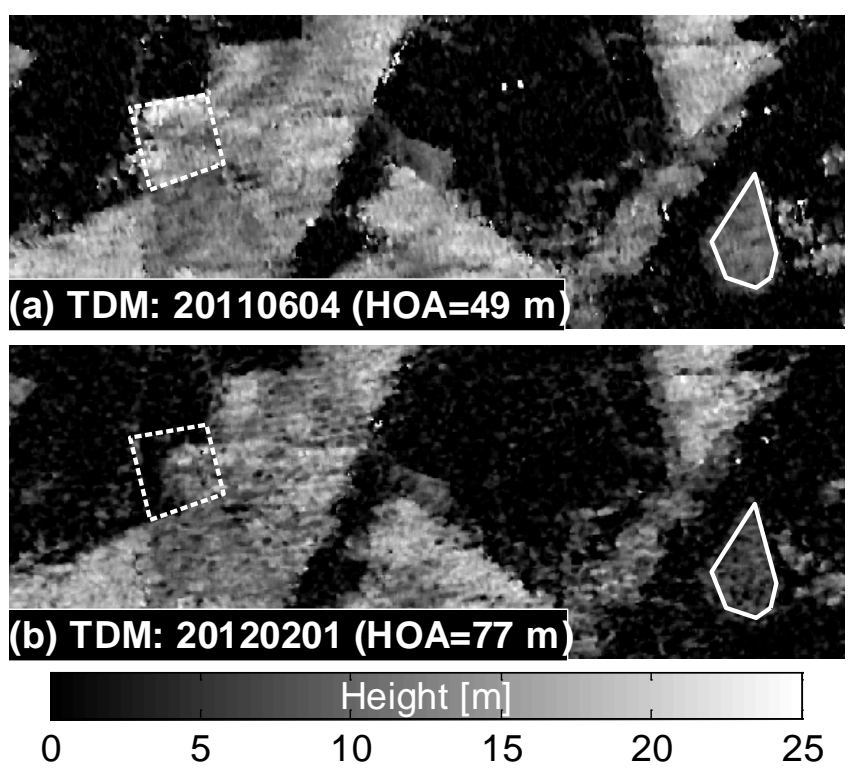

Figure 4 A comparison between two TDM DCMs for a 1600 $\mathrm{m} \times 600 \mathrm{~m}$ region in Remningstorp is here shown. The birch stand discussed in Section 4 is delineated with solid lines. Also, a clear-cut is detected and delineated in dashed lines. VVpolarized TDM data acquired at the nominal angle of incidence of $41^{\circ}$ were used for TDM DCM estimation.

In Figure 2, scatter plots for stand-level TDM height averages are shown. In Figure 2a), a comparison between HOA $=49 \mathrm{~m}$ and HOA=77 $\mathrm{m}$ is shown. The correlation is very high $\left(\mathrm{R}^{2}=0.98\right)$, but a bias can be observed. This can probably be attributed to seasonal changes as the two images are acquired in summer and winter, respectively. In Figure 2b) and Figure 2c), height estimates from an image with HOA $=178 \mathrm{~m}$ are compared with two previously mentioned images with lower $\mathrm{HOA}$ values. When comparing $\mathrm{HOA}=178$ $\mathrm{m}$ and $\mathrm{HOA}=79 \mathrm{~m}$, an offset can be observed in the first image. This can be explained by the uncertainty of absolute height calibration due to the noisiness of the image. In Figure 2d)-f), scatter plots showing temporal stability are shown. Two images separated in time with one repeat cycle (11 days) are compared for three HOA levels: around $50 \mathrm{~m}$, around $80 \mathrm{~m}$, and around $180 \mathrm{~m}$. As it can be observed, correlation is excellent for the lower HOA levels. For the highest HOA value, the aforementioned offset is clearly visible.

In Figure 3 a comparison between three DCMs for a 1600 m x 1000 m region in Krycklan is shown. Lidar DCM was acquired in August 2008, while the two other DCMs were acquired with TDM in August 2011 and 2012, respectively. A few changes can be observed between these acquisitions. These changes can be attributed to clear-cutting done in the forest.

In Figure 4, a comparison between a summer DCM and a winter DCM for a $1200 \mathrm{~m}$ x $1000 \mathrm{~m}$ region in Remningstorp is shown. The two DCMs are in general consistent with each other. The previously mentioned seasonal change can, however, be observed in the delineated deciduous (birch) stand. For this stand, TDM height in the winter DCM is significantly lower than in the summer DCM. This is probably due to the lack of leaves during winter.

\section{CONCLUSIONS}

The approach presented in this paper shows great potential as a future tool for detailed forest mapping. In countries like Sweden, where a high-resolution DEM will be available on a national scale, this method can easily be extended to larger areas. Further work in the project involves derivation of biomass retrieval algorithms based on physical models and regression, modeling of seasonal variations and topographic influence.

\section{ACKNOWLEDGEMENTS}

The authors would like to thank Swedish National Space Board (SNSB) for the funding, German Aerospace Center (DLR) for the TanDEM-X data, Swedish Land Survey for the DEM data, and Swedish Meteorological and Hydrological Institute (SMHI) for the weather data.

\section{REFERENCES}

[1] G. Krieger, A. Moreira, H. Fiedler, I. Hajnsek, M. Werner, M. Younis, et al., "TanDEM-X: A Satellite Formation for High-Resolution SAR Interferometry," IEEE Transactions on Geoscience and Remote Sensing, vol. 45, pp. 3317-3341, 2007.

[2] S. Solberg, R. Astrup, T. Gobakken, E. Naesset, and D. J. Weydahl, "Estimating Spruce and Pine Biomass with Interferometric X-Band SAR," Remote Sensing of Environment, vol. 114, pp. 2353-2360, 2010.

[3] J. Praks, O. Antropov, and M. T. Hallikainen, "LIDARAided SAR Interferometry Studies in Boreal Forest: Scattering Phase Center and Extinction Coefficient at Xand L-Band," Geoscience and Remote Sensing, IEEE Transactions on, vol. 50, pp. 3831-3843, 2012.

[4] M. J. Soja, G. Sandberg, and L. M. H. Ulander, "Regression-Based Retrieval of Boreal Forest Biomass in Sloping Terrain using P-band SAR Backscatter Intensity Data," IEEE Transactions on Geoscience and Remote Sensing, vol. 51, pp. 2646-2665, 2013.

[5] U. Balss, H. Breit, S. Duque, T. Fritz, and C. Rossi, "TanDEM-X Payload Ground Segment: CoSSC Generation and Interferometric Considerations," German Aerospace Center (DLR)2012. 